\title{
Tree Reconstruction via a Closure Operation on Partial Splits *
}

\author{
Charles Semple and Mike Steel \\ Biomathematics Research Centre \\ Department of Mathematics and Statistics \\ University of Canterbury \\ Private Bag 4800 \\ Christchurch, New Zealand \\ c. semple@math.canterbury.ac.nz, m.steel@math. canterbury.ac.nz
}

\begin{abstract}
A fundamental problem in biological classification is the reconstruction of phylogenetic trees for a set $X$ of species from a collection of either subtrees or qualitative characters. This task is equivalent to tree reconstruction from a set of partial $X$-splits (bipartitions of subsets of $X)$. In this paper, we define and analyse a "closure" operation for partial $X$-splits that was informally proposed by Meacham [5]. In particular, we establish a sufficient condition for such an operation to reconstruct a tree when there is essentially only one tree that displays the partial $X$-splits. This result exploits a recent combinatorial result from [2].
\end{abstract}

\section{Introduction}

Trees that have some vertices labelled by elements from a finite set $X$ are often used to represent evolutionary relationships, particularly in biology. Two closely related problems are

(i) determining how to combine such trees that classify overlapping subsets of $X$ into a parent tree that displays each of the input trees, and

(ii) determining how to reconstruct a parent tree from (partial) qualitative characters (equivalently, partitions of $X$ or subsets of $X$ ) so that each character could have evolved on the parent tree without any reverse or convergent transitions (this is equivalent to each tree displaying the partition associated with each character).

For either problem, a parent tree may not exist, and even deciding this turns out to be an NP-complete problem [3, 6]. However, in certain cases, various efficient "rules" for extending sets of trees or sets of characters can either determine

\footnotetext{
^ This work was supported by the New Zealand Marsden Fund (UOC-MIS-003).
} 
that such a tree does not exist, or reconstruct the tree when there is essentially only one possible parent tree. Conditions for such an approach to succeed using extension rules on sets of trees were recently established in [1], using a combinatorial result from [2]. In this paper we take a related, but different, approach by considering a rule for extending sets of partial binary characters (called partial $X$-splits below) that was proposed informally by Meacham [5]. We formalise an iterative construction using this rule, and show that it always leads to the same set of partial $X$-splits, regardless of the possible choices by which the rule can be applied. Then, using the main combinatorial result from [2], we provide sufficient conditions for this construction to successfully recover a parent tree or determine that no such tree exists. Note that although the input to our tree reconstruction problem consists of partial $X$-splits, it could easily be modified to input partitions of subsets of $X$ (in the case of problem (ii)) or trees classifying overlapping subsets of $X$ (in the case of problem (i)) since all these problems are essentially equivalent [6].

\section{Preliminaries}

Throughout this paper, $X$ denotes a finite set. We begin with some definitions.

Partial splits. A partial split of $X$, or more briefly a partial $X$-split, is a partition of a subset of $X$ into two disjoint non-empty subsets. If these two subsets are $A$ and $B$, we denote the partial split by $A \mid B$. Note that no distinction is made between $A \mid B$ and $B \mid A$. If $A \cup B=X$ we say that $A \mid B$ is a (full) $X$-split. We write $a a^{\prime} \mid b b^{\prime}$ to denote the partial split $A \mid B$ if $A=\left\{a, a^{\prime}\right\}$ and $B=\left\{b, b^{\prime}\right\}$, and we call this a quartet $X$-split. We say that the partial split $A^{\prime} \mid B^{\prime}$ extends the partial split $A \mid B$ precisely if either $A \subseteq A^{\prime}$ and $B \subseteq B^{\prime}$ or $A \subseteq B^{\prime}$ and $B \subseteq A^{\prime}$. A partial $X$-split $A \mid B$ is trivial if $\min \{|A|,|B|\}=1$.

$X$-trees. Let $T$ be a tree with vertex set $V$ and edge set $E$, and suppose we have a map $\phi: X \rightarrow V$ with the property that, for all $v \in V$ with degree at most two, $v \in \phi(X)$. Then the ordered pair $(T ; \phi)$, which we frequently denote by $\mathcal{T}$, is called an $X$-tree. Two $X$-trees $\left(T_{1} ; \phi_{1}\right)$ and $\left(T_{2} ; \phi_{2}\right)$, where $T_{1}=\left(V_{1}, E_{1}\right)$ and $T_{2}=\left(V_{2}, E_{2}\right)$, are regarded as equivalent if there exists a bijection $\psi: V_{1} \rightarrow V_{2}$ which induces a bijection between $E_{1}$ and $E_{2}$ and satisfies $\phi_{2}=\psi \circ \phi_{1}$, in which case, $\psi$ is unique.

Let $\mathcal{T}=(T ; \phi)$ be an $X$-tree and let $e$ be an edge of $T$. Then corresponding to $e$ is the $X$-split $\phi^{-1}\left(V_{1}\right) \mid \phi^{-1}\left(V_{2}\right)$, where $V_{1}$ and $V_{2}$ denote the vertex sets of the two components obtained from $T$ by deleting $e$. For an $X$-tree $\mathcal{T}$, let $\Sigma(\mathcal{T})$ (resp. $\Sigma^{*}(\mathcal{T})$ ) denote the collection of non-trivial $X$-splits (resp. all $X$-splits) corresponding to the edges of $T$. 
Compatibility. Let $A \mid B$ be a partial $X$-split. An $X$-tree $\mathcal{T}=(T ; \phi)$ displays $A \mid B$ if there is an edge $e$ of $T=(V, E)$ such that, in $(V, E-\{e\})$, the sets $\phi(A)$ and $\phi(B)$ are subsets of the vertex sets of different components. For example, the $X$-tree shown in Figure 1, where $X=\{1,2, \ldots, 7\}$, displays each of the partial $X$-splits in $\{\{1,2\}|\{3,4\},\{2,3\}|\{4,7\},\{1,7\}|\{4,5\},\{2,5\}|\{6,7\}\}$. A collection $\Sigma$ of partial $X$-splits is said to be compatible if there exists an $X$-tree that displays every $X$-split in $\Sigma$. This is equivalent to requiring that every non-trivial split in $\Sigma$ is extended by a split in $\Sigma(\mathcal{T})$.

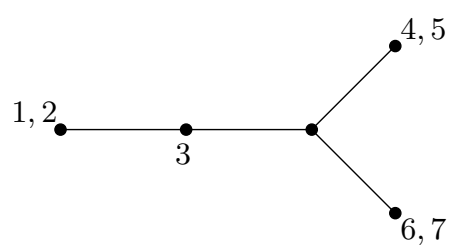

Fig. 1. An $X$-tree displaying $\{1,2\}|\{3,4\},\{2,3\}|\{4,7\},\{1,7\} \mid\{4,5\}$, and $\{2,5\} \mid\{6,7\}\}$.

The following result is well known, and follows immediately from results in [4].

Lemma 1. Let $A_{1} \mid B_{1}$ and $A_{2} \mid B_{2}$ be partial $X$-splits. The following statements are equivalent:

(i) $A_{1} \mid B_{1}$ and $A_{2} \mid B_{2}$ are compatible.

(ii) At least one of the sets $A_{1} \cap A_{2}, A_{1} \cap B_{2}, B_{1} \cap A_{2}$, and $B_{1} \cap B_{2}$ is empty.

A set $\Sigma$ of partial $X$-splits is said to be pairwise compatible if each pair of splits in $\Sigma$ is compatible. This condition is not sufficient for $\Sigma$ to be compatible. For example, for $X=\{a, b, c, d, e\}$, the set $\{a b|c d, a b| c e, a d \mid b e\}$ of partial $X_{-}^{-}$ splits of $X$ is pairwise compatible, but not compatible. However, if $\Sigma$ consists of full $X$-splits, then $\Sigma$ is compatible precisely if $\Sigma$ is pairwise compatible, in which case there is a unique $X$-tree $\mathcal{T}$ such that $\Sigma^{*}(\mathcal{T})=\Sigma$ (see [4]).

Irreducible sets of partial $X$-splits. Let $\Sigma$ be a set of partial splits of $X$. A partial split $A \mid B \in \Sigma$ is redundant if there exists a different partial split in $\Sigma$ that extends $A \mid B$. If $\Sigma$ has no redundant splits, then $\Sigma$ is said to be irreducible. Let $\Sigma_{1}$ and $\Sigma_{2}$ be two irreducible sets of partial splits of $X$. We write $\Sigma_{1} \preceq \Sigma_{2}$ if, for each $A_{1} \mid B_{1} \in \Sigma_{1}$, there is an element $A_{2} \mid B_{2}$ in $\Sigma_{2}$ that extends $A_{1} \mid B_{1}$. It is not difficult to show that $\preceq$ is a partial order on the collection of irreducible sets of partial $X$-splits. Note that if we drop the irreducibility condition, then $\preceq$ may fail to satisfy the antisymmetric property ( $a \preceq b$ and $b \preceq a$ implies $a=b$ ) required of a partial order. Observe that an $X$-tree $\mathcal{T}$ displays a set $\Sigma$ of partial $X$-splits precisely if $\Sigma \preceq \Sigma^{*}(\mathcal{T})$. 
We will let $\mathcal{P}(X)$ denote the collection of all sets $\Sigma$ of partial $X$-splits that are both pairwise compatible and irreducible. Let us adjoin to $\mathcal{P}(X)$ a new element $\omega$, and let $\mathcal{P}_{\omega}(X)=\mathcal{P}(X) \cup\{\omega\}$. If we extend the definition of $\preceq$ by setting $\Sigma \preceq \omega$ for all $\Sigma \in \mathcal{P}(X)$ (so that $\omega$ acts as a maximal element), then $\preceq$ is a partial order on $\mathcal{P}_{\omega}(X)$.

\section{Split Closure}

In this section, we define a "split closure" of a set $\Sigma$ of partial $X$-splits. Informally, we construct an irreducible set of partial $X$-splits from $\Sigma$ by repeatedly applying a pairwise replacement rule along the lines suggested by Meacham [5] (the replacement rule (SC) below corresponds to "Rule 2" in [5]). We then show that any two split closures of $\Sigma$ are equal.

The replacement rule we consider for an irreducible set $\Sigma$ of partial $X$-splits is the following:

(SC) If $A_{1} \mid B_{1}$ and $A_{2} \mid B_{2}$ are elements of $\Sigma$ that satisfy

$$
\emptyset \notin\left\{A_{1} \cap A_{2}, A_{1} \cap B_{2}, B_{1} \cap B_{2}\right\} \text { and } B_{1} \cap A_{2}=\emptyset,
$$

replace $A_{1} \mid B_{1}$ and $A_{2} \mid B_{2}$ in $\Sigma$ by $\left(A_{1} \cup A_{2}\right) \mid B_{1}$ and $A_{2} \mid\left(B_{1} \cup B_{2}\right)$, and then remove any redundant partial splits from the newly created set.

If $A_{1} \mid B_{1}$ and $A_{2} \mid B_{2}$ in the statement of (SC) have the property that $A_{2} \subseteq A_{1}$, $B_{1} \subseteq B_{2}$, and (1) applies, then $B_{1} \cap A_{2}$ is empty, and the two newly created partial splits are $\left(A_{1} \cup A_{2}\right) \mid B_{1}$ and $A_{2} \mid\left(B_{1} \cup B_{2}\right)$, which are identical to $A_{1} \mid B_{1}$ and $A_{2} \mid B_{2}$, respectively. We call such an application of (SC) trivial; in all other (non-trivial) applications of (SC) at least one of the newly created partial splits differs from $A_{1} \mid B_{1}$ or $A_{2} \mid B_{2}$. $\Sigma$.

We say that $\Sigma \in \mathcal{P}(X)$ is closed under (SC) if (SC) applies only trivially to

The motivation for (SC) is the following result due to Meacham [5].

Lemma 2. Let $\Sigma$ be a set of partial $X$-splits, and let $\Sigma^{\prime}$ be a set of partial $X$-splits obtained from $\Sigma$ by a single application of (SC). Then an $X$-tree $\mathcal{T}$ displays $\Sigma$ if and only if $\mathcal{T}$ displays $\Sigma^{\prime}$.

Let $\Sigma$ be a set of irreducible partial $X$-splits, and suppose that we construct a sequence

$$
\Sigma_{0}, \Sigma_{1}, \ldots, \Sigma_{i}, \Sigma_{i+1}, \ldots
$$


of irreducible partial $X$-splits such that $\Sigma_{0}=\Sigma$ and, for all $i \geq 0, \Sigma_{i+1}$ is obtained from $\Sigma_{i}$ by one non-trivial application of (SC) provided $\Sigma_{i}$ is pairwise compatible. Since $\Sigma_{i} \preceq \Sigma_{i+1}$, for all $i \leq 0$, it follows that this sequence is strictly increasing under $\preceq$. Consequently, since the set of all $X$-splits is finite, this sequence must terminate with a set, $\Sigma_{n}$ say, of irreducible partial $X$-splits such that either $\Sigma_{n}$ is pairwise compatible and closed under (SC), or $\Sigma_{n}$ is not pairwise compatible. If the latter holds, we reset $\Sigma_{n}$ to be the element $\omega$.

Definition. We refer to the sequence $\Sigma_{0}, \Sigma_{1}, \ldots, \Sigma_{n}$ as a split closure sequence for $\Sigma$, and the terminal value $\Sigma_{n}$ as a split closure of $\Sigma$. Note that (SC) applies only trivially to $\Sigma_{n}$ (when $\Sigma_{n} \neq \omega$ ) and $\Sigma_{n}$ is always an upper bound, under $\preceq$, to $\Sigma$.

We next provide an explicit bound on the length of any split closure sequence.

Lemma 3. Let $\Sigma$ be a set of irreducible partial $X$-splits, and let $\Sigma_{0}, \Sigma_{1}, \ldots, \Sigma_{n}$ be a split closure sequence for $\Sigma$. Then $n \leq|\Sigma| \times|X|-\sum_{A \mid B \in \Sigma}|A \cup B|$.

Proof. It is straightforward to see that we can prove the lemma by making the additional assumption that $\Sigma_{n} \neq \omega$. For all $i \in\{0,1, \ldots, n-1\}$, let $\lambda_{i}: \Sigma_{i} \rightarrow$ $\Sigma_{i+1}$ be a function that maps an element, $A^{\prime} \mid B^{\prime}$ say, of $\Sigma_{i}$ to an element of $\Sigma_{i+1}$ that extends $A^{\prime} \mid B^{\prime}$. Furthermore, for each element, $A \mid B$ say, of $\Sigma$ and for all $i \in\{0,1, \ldots, n-1\}$, let $A_{i+1} \mid B_{i+1}=\lambda_{i} \lambda_{i-1} \cdots \lambda_{0}(A \mid B)$.

Since, for all $i, \Sigma_{i+1}$ is obtained from $\Sigma_{i}$ by a non-trivial application of (SC) and $\Sigma_{i} \preceq \Sigma_{i+1}$, it follows that

$$
\sum_{A \mid B \in \Sigma}\left(\left|A_{i+1} \cup B_{i+1}\right|-\left|A_{i} \cup B_{i}\right|\right) \geq 1,
$$

for all $i$. Consequently,

$$
\sum_{A \mid B \in \Sigma}\left(\left|A_{n} \cup B_{n}\right|-|A \cup B|\right) \geq n .
$$

Therefore, as $\left|A_{n} \cup B_{n}\right|-|A \cup B| \leq|X|-|A \cup B|$ for each element $A \mid B$ in $\Sigma$,

$$
n \leq|\Sigma| \times|X|-\sum_{A \mid B \in \Sigma}|A \cup B|
$$

as required.

It will immediately follow from Lemma 4 that the split closure of a set $\Sigma$ of irreducible partial $X$-splits is well-defined.

Lemma 4. Let $\Sigma$ be an irreducible set of partial $X-s p l i t s$. Then any two split closures of $\Sigma$ are equal. 
Proof. If every split closure of $\Sigma$ is $\omega$, the lemma is (trivially) true, so we may assume that there exists a split closure, $\bar{\Sigma}$ say, of $\Sigma$ which is not $\omega$. We prove the lemma by showing that every other split closure of $\Sigma$ equals $\bar{\Sigma}$. To this end, let $\Sigma_{0}, \Sigma_{1}, \ldots, \Sigma_{n}$ be a split closure sequence for $\Sigma$, where $\Sigma_{0}=\Sigma$. We first claim that, for all $i \in\{0,1, \ldots, n\}$,

$$
\Sigma_{i} \neq \omega \text { and } \Sigma_{i} \preceq \bar{\Sigma} \text {. }
$$

We establish (2) by induction on $i$. If $i=0$, then (2) holds as there exists a split closure of $\Sigma$ not equal to $\omega$. Now suppose that (2) holds for $i=r$, where $r \in\{0,1, \ldots, n-1\}$, and $\Sigma_{r+1}$ is obtained from $\Sigma_{r}$ by applying (SC) to the pair $A_{1} \mid B_{1}$ and $A_{2} \mid B_{2}$. Without loss of generality, we may assume that $B_{1} \cap A_{2}=\emptyset$. By the induction hypothesis, $\Sigma_{r} \preceq \bar{\Sigma}$, and so there is a pair of partial $X$-splits $A_{1}^{\prime} \mid B_{1}^{\prime}$ and $A_{2}^{\prime} \mid B_{2}^{\prime}$ in $\bar{\Sigma}$ such that $A_{i} \subseteq A_{i}^{\prime}$ and $B_{i} \subseteq B_{i}^{\prime}$, for all $i \in\{1,2\}$. Since $\bar{\Sigma}$ is pairwise compatible, it follows that $A_{1}^{\prime} \mid B_{1}^{\prime}$ and $A_{2}^{\prime} \mid B_{2}^{\prime}$ satisfy (1). Therefore, as (SC) applies only trivially to $\bar{\Sigma}$, it follows that $A_{2}^{\prime} \subseteq A_{1}^{\prime}$ and $B_{1}^{\prime} \subseteq B_{2}^{\prime}$. Consequently, $A_{1}^{\prime} \mid B_{1}^{\prime}$ and $A_{2}^{\prime} \mid B_{2}^{\prime}$ extend $\left(A_{1} \cup A_{2}\right) \mid B_{1}$ and $A_{2} \mid\left(B_{1} \cup B_{2}\right)$, respectively, and so

$$
\Sigma_{r} \cup\left\{\left(A_{1} \cup A_{2}\right)\left|B_{1}, A_{2}\right|\left(B_{1} \cup B_{2}\right)\right\}-\left\{A_{1}\left|B_{1}, A_{2}\right| B_{2}\right\} \preceq \bar{\Sigma} .
$$

Therefore, as $\bar{\Sigma}$ is pairwise compatible, $\Sigma_{r+1} \neq \omega$ and $\Sigma_{r+1} \preceq \bar{\Sigma}$. This completes the induction step and thereby establishes (2).

Applying (2) to $i=n$, we get $\Sigma_{n} \neq \omega$ and $\Sigma_{n} \preceq \bar{\Sigma}$. By interchanging the roles of $\Sigma_{n}$ and $\bar{\Sigma}$ in the argument of the last paragraph, we deduce that $\bar{\Sigma} \preceq \Sigma_{n}$, and hence $\Sigma_{n}=\bar{\Sigma}$.

Definition. In view of Lemma 4 , we denote the split closure of a set $\Sigma$ of irreducible partial $X$-splits by $\operatorname{spcl}(\Sigma)$.

Note that $|\operatorname{spcl}(\Sigma)| \leq|\Sigma|$. Also, provided we set $\operatorname{spcl}(\omega)=\omega$, then $\operatorname{spcl}$ satisfies the three properties one expects of a closure operation on the poset $\mathcal{P}_{\omega}(X)$. Namely, if $a, b \in \mathcal{P}_{\omega}(X)$ with $a \preceq b$, then

(i) $a \preceq \operatorname{spcl}(a)$,

(ii) $\operatorname{spcl}(a) \preceq \operatorname{spcl}(b)$, and

(iii) $\operatorname{spcl}(\operatorname{spcl}(a))=\operatorname{spcl}(a)$.

The next result follows immediately from Lemma 2, however, the converse of this corollary is not true [6].

Corollary 1. Let $\Sigma$ be a set of irreducible partial $X-$ splits. If $\operatorname{spcl}(\Sigma)=\omega$, then $\Sigma$ is incompatible. 


\section{Tree Reconstruction Using Split Closure}

In this section, we establish a sufficient condition for the split closure of an irreducible set of partial $X$-splits to recover all the non-trivial splits of an $X$ tree. This result, Corollary 2, depends on a combinatorial theorem from [2]. In order to apply this theorem, we need to relate partial splits and split closure to quartet splits and a dyadic closure rule that operates on quartet splits. To this end, we introduce some further definitions.

Definition. For a partial $X$-split $A \mid B$, let

$$
\mathcal{Q}(A \mid B)=\left\{a a^{\prime} \mid b b^{\prime}: a, a^{\prime} \in A ; b, b^{\prime} \in B ; a \neq a^{\prime} ; \text { and } b \neq b^{\prime}\right\}
$$

and, for a set $\Sigma$ of partial $X$-splits, let

$$
\mathcal{Q}(\Sigma)=\bigcup_{A \mid B \in \Sigma} \mathcal{Q}(A \mid B)
$$

For an $X$-tree $\mathcal{T}$, we denote $\mathcal{Q}(\Sigma(\mathcal{T}))$ by $\mathcal{Q}(\mathcal{T})$.

Proposition 1 relates partial splits to quartet splits.

Proposition 1. Let $\Sigma$ be an irreducible set of non-trivial partial $X$-splits and let $\mathcal{T}$ be an $X$-tree. Then $\Sigma=\Sigma(\mathcal{T})$ if and only if the following two conditions hold:

(i) $|\Sigma| \leq|\Sigma(\mathcal{T})|$; and

(ii) $\mathcal{Q}(\Sigma)=\mathcal{Q}(\mathcal{T})$.

Proof. Evidently, if $\Sigma=\Sigma(\mathcal{T})$, then (i) and (ii) hold. For the converse, we first show that $\Sigma \preceq \Sigma(\mathcal{T})$. Let $\mathcal{T}=(T ; \phi)$, and let $A \mid B$ be an element of $\Sigma$. By (ii), $\mathcal{Q}(A \mid B) \subseteq \mathcal{Q}(\mathcal{T})$. Therefore, for each quartet of elements $a, a^{\prime}, b$, and $b^{\prime}$ with $a, a^{\prime} \in \bar{A}$ and $b, b^{\prime} \in B$, the cardinality, denoted $n\left(a, a^{\prime}, b, b^{\prime}\right)$, of $\left\{A^{\prime}\left|B^{\prime} \in \Sigma(\mathcal{T}): a a^{\prime}\right| b b^{\prime} \in \mathcal{Q}\left(A^{\prime} \mid B^{\prime}\right)\right\}$ satisfies $n\left(a, a^{\prime}, b, b^{\prime}\right) \geq 1$. Now suppose that $a, a^{\prime}, b$, and $b^{\prime}$ are chosen so that $n\left(a, a^{\prime}, b, b^{\prime}\right)$ is minimised, and $A^{\prime} \mid B^{\prime}$ is an element of $\Sigma(\mathcal{T})$ with $a a^{\prime} \mid b b^{\prime} \in \mathcal{Q}\left(A^{\prime} \mid B^{\prime}\right)$. By considering the placement of the vertices $\phi(a), \phi\left(a^{\prime}\right), \phi(b)$, and $\phi\left(b^{\prime}\right)$ in $T$, we see that $A \subseteq A^{\prime}$ and $B \subseteq B^{\prime}$, thus showing that $\Sigma \preceq \Sigma(\mathcal{T})$.

Now let $n(A \mid B)=\min \left\{n\left(a, a^{\prime}, b, b^{\prime}\right): a, a^{\prime} \in A ; b, b^{\prime} \in B\right\}$, and let

$$
\Sigma_{1}=\{A \mid B \in \Sigma: n(A \mid B)=1\} .
$$

Using the fact that $\Sigma \preceq \Sigma(\mathcal{T})$, it is easily seen that, for each element, $A \mid B$ say, of $\Sigma_{1}$, there is a unique element of $\Sigma(\mathcal{T})$ that extends $A \mid B$. Let $\mu: \Sigma_{1} \rightarrow \Sigma(\mathcal{T})$ 
denote the map that associates with each element $A \mid B$ of $\Sigma_{1}$ the unique element of $\Sigma(\mathcal{T})$ that extends $A \mid B$. We next show that $\mu$ is a bijection.

Let $C^{\prime} \mid D^{\prime}$ be an element of $\Sigma(\mathcal{T})$, and choose elements $c, c^{\prime} \in C^{\prime}$ and $d, d^{\prime} \in$ $D^{\prime}$ so that $n\left(c, c^{\prime}, d, d^{\prime}\right)=1$. Then, by (ii), there is an element $C \mid D$ of $\Sigma_{1}$ such that $c c^{\prime} \mid d d^{\prime} \in \mathcal{Q}(C \mid D)$ and, moreover, $\mu(C \mid D)=C^{\prime} \mid D^{\prime}$. Thus the map $\mu$ is surjective and so $\left|\Sigma_{1}\right| \geq|\Sigma(\mathcal{T})|$. It now follows from (i) that $\Sigma_{1}=\Sigma$, and so $\mu$ is indeed a bijection. Hence $|\Sigma|=|\Sigma(\mathcal{T})|$. Since $\Sigma_{1}=\Sigma$, we can complete the proof by showing that, for each $A\left|B \in \Sigma_{1}, \mu(A \mid B)=A\right| B$.

Suppose, to the contrary, that $\mu(A \mid B)=A^{\prime} \mid B^{\prime}$, where $A^{\prime} \mid B^{\prime}$ extends $A \mid B$ but is not equal to $A \mid B$, for some $A \mid B \in \Sigma_{1}$. Then there is an element $x$ in $\left(A^{\prime} \cup B^{\prime}\right)-(A \cup B)$. Without loss of generality, we may assume $x \in A^{\prime}$. Then we can choose elements $a_{1} \in A^{\prime}$ and $b_{1}, b_{2} \in B^{\prime}$ so that $n\left(x, a_{1}, b_{1}, b_{2}\right)=1$. By (ii), there is an element $C \mid D$ of $\Sigma_{1}$ such that $x a_{1} \mid b_{1} b_{2} \in \mathcal{Q}(C \mid D)$. Since $x \notin A \cup B$, $C \mid D$ is not equal to $A \mid B$. Therefore, as $\mu$ is a bijection, $\mu(C \mid D) \neq A^{\prime} \mid B^{\prime}$, and so $x a_{1} \mid b_{1} b_{2} \notin \mathcal{Q}(\mu(C \mid D))$. This contradiction completes the proof of Proposition 1.

Following [1], the semi-dyadic closure of a collection $\mathcal{Q}$ of quartet $X$-splits, denoted $\operatorname{scl}_{2}(\mathcal{Q})$, is the minimal set of quartet $X$-splits that contains $\mathcal{Q}$ and is closed under the following rule:

(SDC) If $a b \mid c d$ and $a c \mid d e$ are elements of $\operatorname{scl}_{2}(\mathcal{Q})$, then $a b|c e, a b| d e$, and $b c \mid d e$ are elements of $\operatorname{scl}_{2}(\mathcal{Q})$.

The next proposition relates split closure to semi-dyadic closure.

Proposition 2. If $\Sigma$ is a set of compatible irreducible partial $X$-splits, then $\operatorname{scl}_{2}(\mathcal{Q}(\Sigma)) \subseteq \mathcal{Q}(\operatorname{spcl}(\Sigma))$.

Proof. We can obtain $\operatorname{scl}_{2}(\mathcal{Q}(\Sigma))$ by constructing a sequence $\mathcal{Q}_{0}, \mathcal{Q}_{1}, \ldots, \mathcal{Q}_{m}$ of collections of quartet $X$-splits such that $\mathcal{Q}_{0}=\mathcal{Q}(\Sigma), \mathcal{Q}_{m}=\operatorname{scl}_{2}(\mathcal{Q}(\Sigma))$, and, for all $i \in\{0,1, \ldots, m-1\}, \mathcal{Q}_{i+1}=\mathcal{Q}_{i} \cup \operatorname{scl}_{2}\left(\left\{q_{i}, q_{i}^{\prime}\right\}\right)$, where $q_{i}, q_{i}^{\prime} \in \mathcal{Q}_{i}$ but $\operatorname{scl}_{2}\left(\left\{q_{i}, q_{i}^{\prime}\right\}\right) \nsubseteq \mathcal{Q}_{i}$. We prove the proposition by showing that one can construct a sequence $\Sigma_{0}, \Sigma_{1}, \ldots, \Sigma_{m}$ of sets of irreducible partial $X$-splits such that $\Sigma_{0}=\Sigma$ and, for all $j \in\{0,1, \ldots, m\}$,

$$
\Sigma_{j} \preceq \operatorname{spcl}(\Sigma) \text { and } \mathcal{Q}_{j} \subseteq \mathcal{Q}\left(\Sigma_{j}\right) .
$$

For then, taking $j=m$, establishes the proposition.

The proof of the latter construction is by induction on $j$. Clearly, the result holds if $j=0$ as $\Sigma_{0}=\Sigma$. Now let $r$ be an element of $\{0,1, \ldots, m-1\}$, and 
suppose that $\Sigma_{j}$ has been defined for all $j \leq r$ and (3) holds for $j=r$. Then $q_{r}, q_{r}^{\prime} \in \mathcal{Q}\left(\Sigma_{r}\right)$. If $\operatorname{scl}_{2}\left(\left\{q_{r}, q_{r}^{\prime}\right\}\right) \subseteq \mathcal{Q}\left(\Sigma_{r}\right)$, then set $\Sigma_{r+1}=\Sigma_{r}$. On the other hand, suppose that $\operatorname{scl}_{2}\left(\left\{q_{r}, q_{r}^{\prime}\right\}\right) \nsubseteq \mathcal{Q}\left(\Sigma_{r}\right)$. Since $\mathcal{Q}_{r} \subseteq \mathcal{Q}\left(\Sigma_{r}\right)$, there are two distinct elements $A \mid B$ and $A^{\prime} \mid B^{\prime}$ in $\Sigma_{r}$ that extend $q_{r}$ and $q_{r}^{\prime}$, respectively. As $\Sigma$ is compatible, $\operatorname{spcl}(\Sigma)$ is compatible, so $\Sigma_{r}$ is pairwise compatible. It now follows that we can apply (SC) to $A \mid B$ and $A^{\prime} \mid B^{\prime}$. Set $\Sigma_{r+1}$ to be the resulting set of irreducible partial $X$-splits. In both cases, $\Sigma_{r+1} \preceq \operatorname{spcl}(\Sigma)$ and, moreover, one can easily check that $\operatorname{scl}_{2}\left(\left\{q_{r}, q_{r}^{\prime}\right\}\right) \subseteq \mathcal{Q}\left(\Sigma_{r+1}\right)$. Hence (3) holds for $j=r+1$, and so we can indeed construct such a sequence.

Definition. A set $\Sigma$ of non-trivial partial $X$-splits weakly defines an $X$-tree $\mathcal{T}$ if there is a unique $X$-tree $\mathcal{T}^{\prime}$ that displays $\Sigma \cup\{\{x\} \mid X-\{x\}: x \in X\}$, in which case $\Sigma^{*}(\mathcal{T})=\Sigma\left(\mathcal{T}^{\prime}\right)$.

Let $\mathcal{T}=(T ; \phi)$ be an $X$-tree, and let $v$ be a vertex of $T$. Suppose that there is a set of non-trivial partial $X$-splits that weakly defines $\mathcal{T}$. Then it is easily seen that each of the following hold in $T$ :

(i) If $v$ is a pendant vertex, then $\left|\phi^{-1}(v)\right|=2$.

(ii) If $v$ is a degree-two vertex, then $\left|\phi^{-1}(v)\right|=1$.

(iii) If $v$ is neither a pendant vertex nor a degree-two vertex, then $v$ is a degreethree vertex and $\phi^{-1}(v)=\emptyset$.

Conversely, if $\mathcal{T}$ satisfies all of (i)-(iii), then $\Sigma(\mathcal{T})$ weakly defines $\mathcal{T}$. As an example, the set $\{\{1,2\}|\{3,4\},\{2,3\}|\{4,7\},\{1,7\}|\{4,5\},\{2,5\}|\{6,7\}\}$ of partial $X$-splits weakly defines the $X$-tree in Figure 1 .

Two characterisations for when a minimum-sized set of quartet $X$-splits weakly defines an $X$-tree are given in [2]. Theorem 1 gives a third such characterisation. Before stating this theorem, we note that it immediately follows from [6, Proposition 6] that $|X|-3$ is the minimum number of quartet $X$-splits that can weakly define an $X$-tree $\mathcal{T}$. Observe that $|X|-3=|\Sigma(\mathcal{T})|$.

Theorem 1. Let $\Sigma_{Q}$ be a set of $|X|-3$ quartet $X$-splits, and let $\mathcal{T}$ be an $X$-tree. Then the following statements are equivalent:

(i) $\Sigma_{Q}$ weakly defines $\mathcal{T}$.

(ii) $\operatorname{spcl}\left(\Sigma_{Q}\right)=\Sigma(\mathcal{T})$.

Proof. If $\operatorname{spcl}\left(\Sigma_{Q}\right)=\Sigma(\mathcal{T})$, then one can easily check using Lemma 2 that $\Sigma_{Q}$ weakly defines $\mathcal{T}$. For the converse, suppose that $\Sigma_{Q}$ weakly defines $\mathcal{T}$. Then, by [2, Theorem 3.11] (also see [1]), $\operatorname{scl}_{2}\left(\Sigma_{Q}\right)=\mathcal{Q}(\mathcal{T})$. Since $\Sigma_{Q}$ is compatible and irreducible, we can apply Proposition 2 to $\Sigma_{Q}$ and get $\operatorname{scl}_{2}\left(\mathcal{Q}\left(\Sigma_{Q}\right)\right) \subseteq$ 
$\mathcal{Q}\left(\operatorname{spcl}\left(\Sigma_{Q}\right)\right)$. As $\mathcal{Q}\left(\Sigma_{Q}\right)=\Sigma_{Q}$, it follows that $\mathcal{Q}(\mathcal{T}) \subseteq \mathcal{Q}\left(\operatorname{spcl}\left(\Sigma_{Q}\right)\right)$. Now $\operatorname{spcl}\left(\Sigma_{Q}\right)$ is compatible, so $\mathcal{Q}(\mathcal{T})=\mathcal{Q}\left(\operatorname{spcl}\left(\Sigma_{Q}\right)\right)$. Moreover, $\left|\operatorname{spcl}\left(\Sigma_{Q}\right)\right| \leq\left|\Sigma_{Q}\right|=$ $|\Sigma(\mathcal{T})|$, and so, by Proposition $1, \operatorname{spcl}\left(\Sigma_{Q}\right)=\Sigma(\mathcal{T})$ as required.

An immediate consequence of Theorem 1 is Corollary 2.

Corollary 2. Let $\Sigma_{Q}$ be a set of quartet $X$-splits, and suppose that there exists a subset of $\Sigma_{Q}$ of size $|X|-3$ that weakly defines an $X$-tree $\mathcal{T}$. If $\Sigma_{Q}$ is compatible, then $\operatorname{spcl}\left(\Sigma_{Q}\right)=\Sigma(\mathcal{T})$; otherwise $\operatorname{spcl}\left(\Sigma_{Q}\right)=\omega$.

Suppose that $\Sigma_{Q}$ and $\mathcal{T}$ satisfy the assumptions of their namesake in the statement of Corollary 2. The potential utility of Corollary 2 lies in the fact that $\mathcal{T}$ can be reconstructed from $\Sigma(\mathcal{T})$ and, in turn, $\Sigma(\mathcal{T})=\operatorname{spcl}\left(\Sigma_{Q}\right)$ can be reconstructed from $\Sigma_{Q}$; moreover, both tasks can be carried out in polynomial time. Thus we obtain an alternative polynomial-time algorithm for the special case of this tree reconstruction problem to that described in [1]. Furthermore, if $\left|\Sigma_{Q}\right|=O(n)$, then, by Lemma 3, every split closure sequence for $\Sigma_{Q}$ has length at most $O\left(n^{2}\right)$, and so the algorithm described here should be reasonably fast.

\section{Acknowledgements}

We thank the anonymous referee for their helpful comments.

\section{References}

[1] Böcker, S., Bryant, D., Dress, A. W. M., and Steel, M. A.: Algorithmic aspects of tree amalgamation. To appear in Journal of Algorithms.

[2] Böcker, S., Dress, A. W. M., and Steel, M.: Patching up X-trees. Annals of Combinatorics 3 (1999) 1-12.

[3] Bodlaender, H. L., Fellows, M. R., and Warnow, T. J.: Two strikes against perfect phylogeny. In Proceedings of the International Colloquium on Automata, Languages and Programming, Vol. 623 of Lecture Notes in Computer Science, Springer-Verlag, Berlin (1993) 273-283.

[4] Buneman, P.: The recovery of trees from measures of dissimilarity. In F. R. Hodson, D. G. Kendall, and P. Tautu (eds.): Mathematics in the Archaeological and Historical Sciences, Edinburgh University Press (1971) 387-395.

[5] Meacham, C. A.: Theoretical and computational considerations of the compatibility of qualitative taxonomic characters. In J. Felsenstein (ed.): Numerical Taxonomy, NATO ASI Series Vol. G1, Springer-Verlag (1983) 304-314.

[6] Steel, M.: The complexity of reconstructing trees from qualitative characters and subtrees. Journal of Classification 9(1) (1992) 91-116. 